\title{
Hybridization may facilitate in situ survival of endemic species through periods of climate change
}

\author{
Matthias Becker ${ }^{1 \star}$, Nicole Gruenheit ${ }^{1,2}$, Mike Steel $^{3}$, Claudia Voelckel ${ }^{1}$, Oliver Deusch ${ }^{1}$, \\ Peter B. Heenan ${ }^{4}$, Patricia A. McLenachan ${ }^{1}$, Olga Kardailsky ${ }^{5}$, Jessica W. Leigh ${ }^{6}$ \\ and Peter J. Lockhart ${ }^{1}$
}

\begin{abstract}
Predicting survival and extinction scenarios for climate change requires an understanding of the present day ecological characteristics of species and future available habitats, but also the adaptive potential of species to cope with environmental change. Hybridization is one mechanism that could facilitate this. Here we report statistical evidence that the transfer of genetic information through hybridization is a feature of species from the plant genus Pachycladon that survived the Last Glacial Maximum in geographically separated alpine refugia in New Zealand's South Island. We show that transferred glucosinolate hydrolysis genes also exhibit evidence of intralocus recombination. Such gene exchange and recombination has the potential to alter the chemical defence in the offspring of hybridizing species. We use a mathematical model to show that when hybridization increases the adaptive potential of species, future biodiversity will be best protected by preserving closely related species that hybridize rather than by conserving distantly related species that are genetically isolated.
\end{abstract}

Predicting the response of organisms and estimating loss of genetic diversity are important challenges for evaluating the impact of global climate change on biodiversity ${ }^{1-3}$. Although ecological modelling has an important place in understanding this impact ${ }^{2}$, an accurate prediction of range shift and extinction of species also requires determining their adaptive potential, and in particular the frequency with which hybridization facilitates adaptation ${ }^{4}$. Determining this is important, because although hybridization can be a maladaptive phenomenon ${ }^{5}$, it might also help species to acquire adaptive traits, respond successfully to environmental change and invade new habitats ${ }^{1,3,4,6-8}$. A better understanding of its positive and negative contributions is essential for evaluating biodiversity impacts.

Species affected by climate change in the past-the consequences of which are manifested in extant species' ranges and patterns of genetic diversity-provide models to test for signatures of hybridization. Here we studied Pachycladon (Brassicaceae), an allopolyploid genus of 11 species $^{9}$ that have radiated in the New Zealand Alps during the Pleistocene period ${ }^{9,10}$. Figure 1 shows ice cover at the height of the Last Glacial Maximum (LGM; 21,00018,000 years ago), the present day distribution of three Pachycladon species, and a chloroplast TCS (statistical parsimony) haplotype network indicating relationships among accessions of the three species. At present, all three species are restricted to greywacke rock in the central and northern regions of the South Island of New
Zealand ${ }^{9}$. On the basis of ecological characteristics and prediction of available habitats, it has been proposed that there was nunatak survival in the central South Island at the height of the LGM for Pachycladon enysii, and survival in more northern and peripheral South Island refugia for $P$. fastigiatum and $P$. stellatum ${ }^{9}$.

However, hybridization and the introgression of adaptively significant traits between species could challenge our understanding of this scenario and other similar survival scenarios for biodiversity ${ }^{2}$ that are based on present day species' attributes and present habitats. To investigate this with Pachycladon, we tested for evidence of past introgression with chloroplast markers from five loci (Supplementary Fig. 1) in accessions sampled across the geographic ranges of $P$. enysii, $P$. fastigiatum and $P$. stellatum using the JML (Joly, McLenachan, Lockhart) test of ref. 11 and a species tree reconstructed from seven nuclear gene loci (Supplementary Fig. 2).

We found that incomplete lineage sorting was unable to explain identical and highly similar chloroplast haplotypes shared by $P$. enysii, $P$. fastigiatum and $P$. stellatum accessions (Fig. 1 and Supplementary Fig. 3). At probability value $p<0.1$ there was evidence for introgression of chloroplast genomes between adjacent central populations of $P$. enysii and $P$. fastigiatum and between adjacent northern populations of $P$. enysii and $P$. stellatum (Supplementary Fig. 3 and Table 4). This suggests that there has been regional chloroplast capture through sympatric events of hybridization.

Relaxed molecular clock estimates (Supplementary Fig. 4) indicate that the chloroplast haplotypes of the central populations of $P$. enysii and $P$. fastigiatum diverged from the haplotypes of northern populations at least 190,000 years ago (95\% highest posterior density lower bound: 0.19 million years ago (Ma) $-95 \%$ highest posterior density upper bound: $1 \mathrm{Ma}$ ). In contrast, analyses of nuclear molecular markers identify identical or highly similar alleles common to northern and central populations of Pachycladon species. Thus, although restricted seed dispersal and in situ survival of central populations of Pachycladon through successive glacial-interglacial cycles over the Pleistocene seems likely, central and northern populations also seem to have been connected by pollenmediated gene flow.

In general, species boundaries seem to be maintained between $P$. enysii, $P$. fastigiatum, and P. stellatum. This is suggested from Bayesian delimitation analysis for seven nuclear genes (S. Joly, P. B. Heenan and P. J. Lockhart, manuscript in preparation).

\footnotetext{
${ }^{1}$ Institute of Fundamental Sciences, Massey University, Palmerston North 4442, New Zealand, ${ }^{2}$ Faculty of Life Sciences, University of Manchester, Oxford Road, Manchester M13 9PP, UK, ${ }^{3}$ Allan Wilson Centre, University of Canterbury, Christchurch 8140, New Zealand, ${ }^{4}$ Landcare Research, Lincoln 7640 , New Zealand, ${ }^{5}$ Department of Anatomy, University of Otago, Dunedin 9054, New Zealand, ${ }^{6}$ Department of Mathematics and Statistics, University of Otago, Dunedin 9054, New Zealand. *e-mail:m.becker@massey.ac.nz
} 


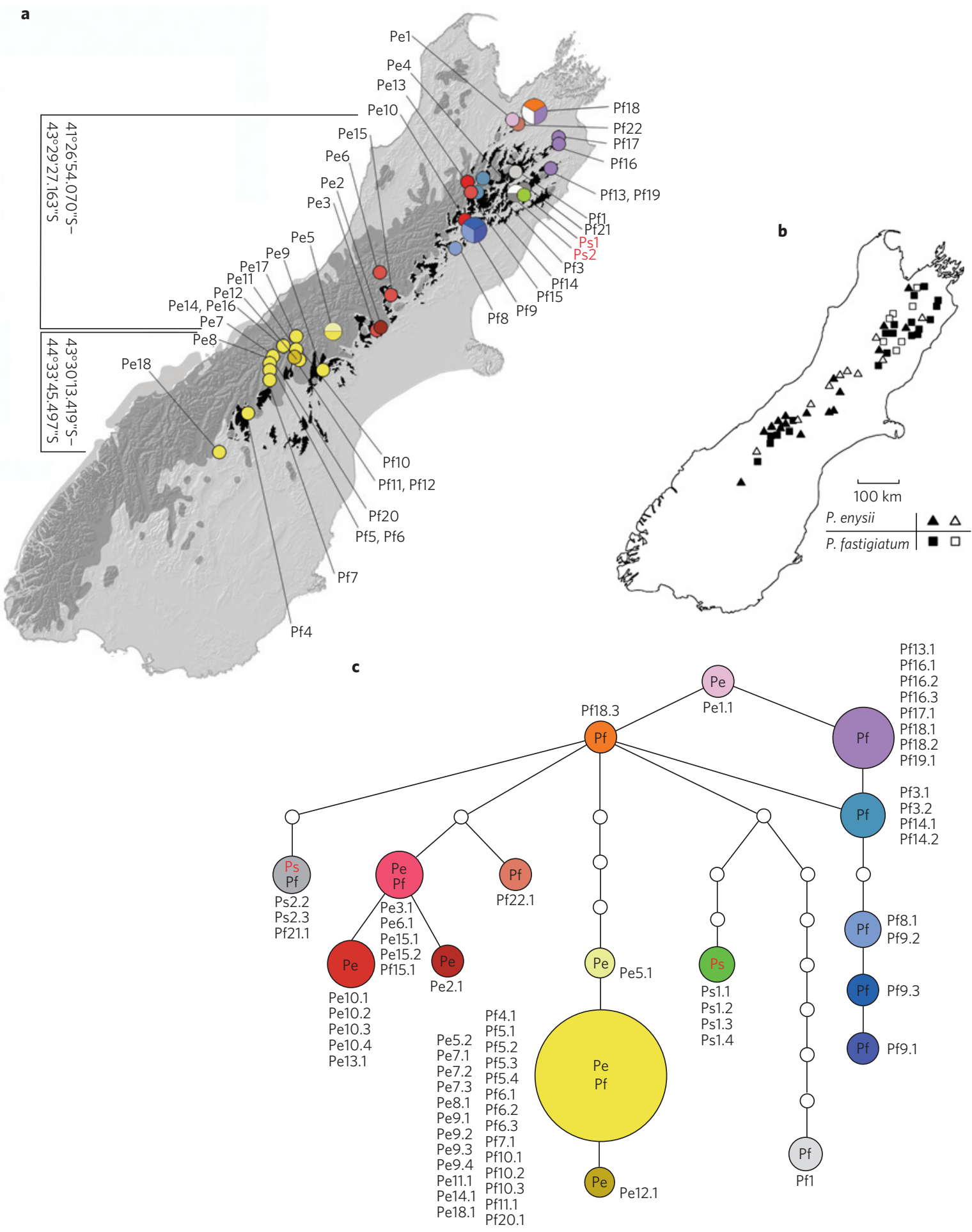

Figure 1 | Phylogeographic distribution of $P$. enysii (Pe), P. fastigiatum (Pf) and P. stellatum (Ps) populations. a, Ice cover (dark grey) and peripheral refugia (black) at the height of the LGM. Coordinates for range limits of haplotype clusters are given. Haplotypes are colour coded; multi-coloured circles identify populations (for example, Pf18) with multiple genotypes (for example, Pf 18.1-4). b. Sampling performed across distribution areas of $P$. enysii and $P$. fastigiatum: filled shapes represent sampled populations and open shapes represent unsampled populations ( $P$. stellatum not shown as it occurs only in two adjacent locations, Fig. 1a and Supplementary Table 1). c, TCS network of relationships among $P$. enysii, $P$. fastigiatum, and $P$. stellatum chloroplast haplotypes.

However, despite this finding there is also evidence for introgression of nuclear genes of potential adaptive significance between these species (Fig. 2). These are the epithiospecifier modifier (ESM1; ref. 12), epithiospecifier protein (ESP; ref. 13), and modified vacuole phenotype1 (MVP1; ref. 14) genes whose differential expression is associated with species-specific glucosinolate hydrolysis phenotypes in Pachycladon ${ }^{15}$ and which, in Arabidopsis thaliana, have been demonstrated to differentially affect herbivore fitness ${ }^{16}$. The expressed alleles of ESP and ESM1 showed strong signatures of positive and purifying selection in Pachycladon (Fig. 3). JML 

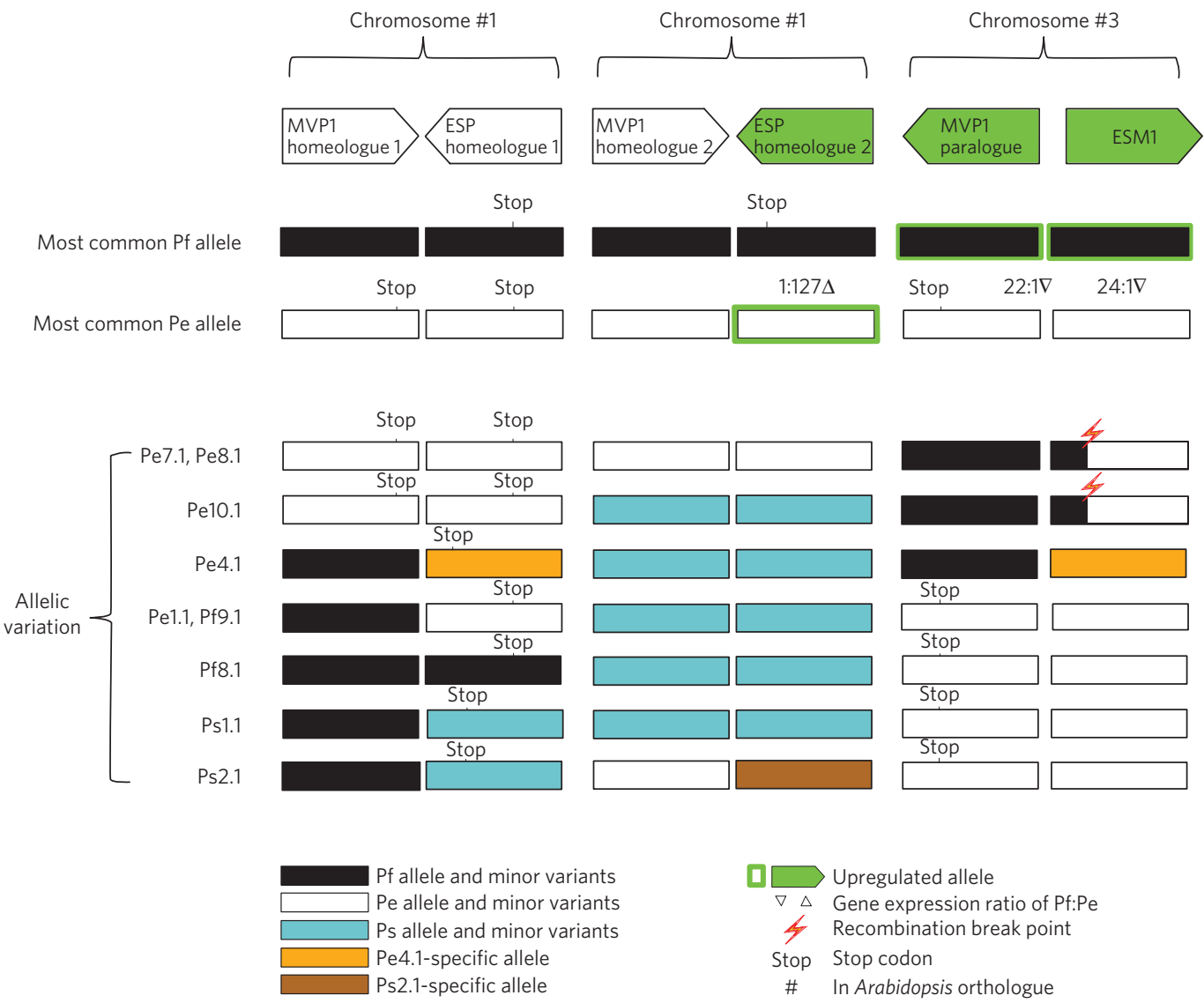

Figure 2 | Recombination and allelic variants of glucosinolate hydrolysis genes in Pachycladon. Alleles shared between P. enysii, P. fastigiatum and P. stellatum were found for six loci implicated in glucosinolate hydrolysis: two homeologues and one paralogue of MVP1, two homeologues of ESP and a single ESM1 gene. Allele sharing due to recent introgression was confirmed for MVP1 homeologue 2, ESP homeologue 2 and ESM1. Recombination within ESM1 of $P$. enysii and $P$. fastigiatum led to introgression of the $5^{\prime}$ exon region of the $P$. fastigiatum ESM1 allele and the neighbouring MVP1 paralogue allele into accessions of $P$. enysii (for a detailed discussion of these results refer to Supplementary Information 1).

(ref. 11) identified alleles of glucosinolate hydrolysis genes-MVP1 homeologue 2, ESP homeologue 2 and ESM1 - to be introgressed between northern populations of $P$. enysii, $P$. fastigiatum and $P$. stellatum (Supplementary Table 5). Allele sharing between species is also observed with the other glucosinolate hydrolysis loci under investigation (MVP1 homeologue 1, ESP homeologue 1 and MVP1 paralogue; Fig. 2). However, this observation is more difficult to assess because of low sequence variation. In the instances of statistically inferred introgression, allele sharing has altered the functional allelic diversity of populations in all three species of Pachycladon (Supplementary Tables 6 and 7). Furthermore, a likelihood ratio test indicates that intra-genic recombination has occurred within the ESM1 locus in P. enysii (Supplementary Fig. 5; $\left.p=4.7 \times 10^{-11}\right)$. This hybridization-mediated recombination has replaced a neighbouring MVP1 paralogue allele, characterized by a frame shift and premature stop codon, with an allele that has an open reading frame and is expressed ${ }^{17}$ in P. fastigiatum. This modification alters the functional allelic diversity of the MVP1 paralogue locus in P. enysii (Fig. 2).

These above instances of introgression and recombination, are likely to impact on glucosinolate hydrolysis chemotypes (Supplementary Table 6), herbivore fitness and the adaptive potential of Pachycladon species. In contrast to the chloroplast results, introgression of glucosinolate hydrolysis genes is not restricted to geographically adjacent/sympatric populations: The same recombination within ESM1 was found in northern (Pe10) and central populations of $P$. enysii (Pe7, Pe8). Thus, it seems that pollen-mediated nuclear gene flow has dispersed glucosinolate hydrolysis genotypes more widely and facilitated genetic exchange between disjunct populations.

Hybridization is increasingly speculated as an important mechanism for rapid adaptation to environmental change $e^{1,3,4,7}$. However, presumably because of uncertainty in its possible outcomes ${ }^{4}$, hybridization is yet to be incorporated into models that predict the survival and extinction scenarios of biota. Previously, it was suggested ${ }^{9}$ that $P$. enysii survived Pleistocene glaciation in central South Island nunataks, whereas $P$. fastigiatum was exterminated from such areas. However, here we show that introgression between these species is a feature of populations that have persisted in the central part of the South Island of New Zealand over periods of Pleistocene climate change when Pachycladon species ranges would have expanded and contracted with available habitat and changes in biotic interactions. Furthermore, our results suggest that introgression and recombination are mechanisms that have the potential in Pachycladon to rapidly generate adaptive chemotypes important in herbivory and pathogen defence ${ }^{15,16}$. Thus, if introgression and recombination have similarly acted on other adaptive gene sets, they are likely to have aided survival of Pachycladon species in the past and will impact on their adaptive potential in the future. This hypothesis is important to test given recent observations suggesting the adaptive significance of hybridization in animal ${ }^{8,18,19}$ and plant species $^{6}$, as well as interest in determining what processes are most important in shaping ecological interactions ${ }^{20}$.

The suggestion that hybridization and introgression might act as a general mechanism for increasing the adaptive potential of species has implications for predicting and maximizing future 


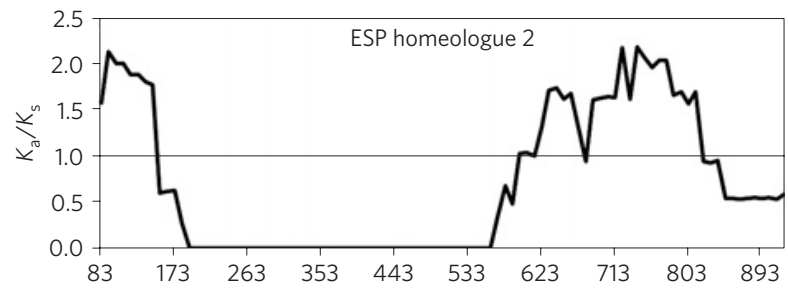

bp

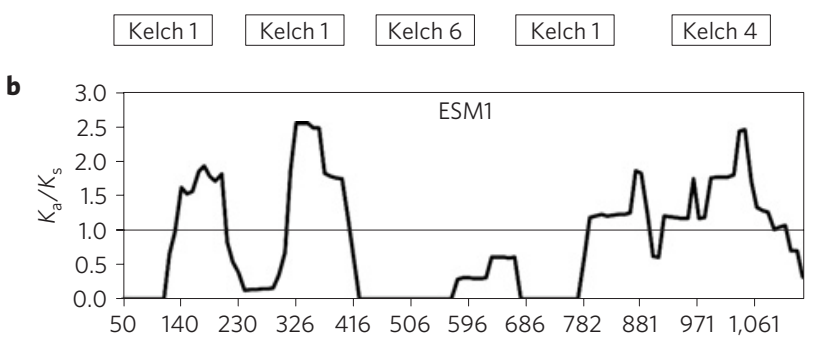

bp

GDSL-like lipase/acylhydrolase

Figure 3 | Selection signatures in open reading frames for Pachycladon ESP (homeologue 2) and ESM1. a, Comparison of ESP homeologue 2 from $P$. enysii and $P$. stellatum. $\mathbf{b}$, Comparison of ESM1 from P. enysii and P. fastigiatum. Domains of ESP homeologue 2 (Kelch 1, 4, and 6, respectively) and ESM1 (GDSL-like lipase/acylhydrolase) confidently predicted by Pfam (http://pfam.sanger.ac.uk/) contain signatures for positive and purifying selection $\left(K_{a} / K_{s}\right.$ ratios $\neq 1$, where $K_{a} / K_{s}$ is the ratio of the number of non-synonymous substitutions per non-synonymous site $\left(K_{\mathrm{a}}\right)$ to the number of synonymous substitutions per synonymous site $\left(K_{s}\right)$ ). These observations are consistent with a hypothesis that glucosinolate hydrolysis genes comprise adaptive gene sets for plant defence in the Brassicaceae ${ }^{13}$.

phylogenetic diversity through conservation efforts ${ }^{21}$. Although hybrids and hybridization have a long and uncomfortable history in conservation biology 22,23 , the preservation and protection of hybrids and/or individuals with the potential to hybridize in some cases could lead to greater preservation of future phylogenetic and biological diversity. We illustrate this conjecture with a simple example.

Suppose there are two groups of closely related species (A, B) and (C, D) that are phylogenetically distant from each other (Fig. 4). The species within each pair occupy overlapping geographic areas. Assume that the probability $p$ of any of these species surviving environmental change in situ is close to zero, but that with conservation intervention we are able to increase the probability of survival of any one species. We will assume that our resources (budget) allow only two species to be conserved. The Noah's Ark problem $^{24}$ in this simple setting is to decide which two species should be preserved, where the aim is to increase expected future phylogenetic diversity.

Ignoring for the moment the influence of hybridization, it is well known that the pair of species having maximal phylogenetic diversity (PD) would have maximal separation in the tree (for example species $\mathrm{A}$ and $\mathrm{C}$ in Fig. 4 , Scenario 1). In this case the expected future $\mathrm{PD}$ is precisely $2 p T$, when the time from the ancestor to the present is $T$ and the length of the four short pendant edges is ignored. In contrast, conserving $\mathrm{A}$ and $\mathrm{B}$ (Scenario 2 ) leads to a future expected $\mathrm{PD}$ value of $\left(2 p-p_{\mathrm{AB}}\right) T$, where $p_{\mathrm{AB}}$ is the probability that both $A$ and $B$ survive ( $p_{\mathrm{AB}}$ would be $p^{2}$ if these events are independent, but as A and B are closely related it will probably be closer to $p$ ). Scenario 1 therefore always leads to higher PD than Scenario 2 in this simple setting.
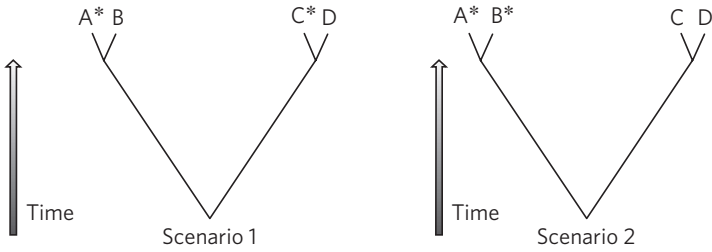

Figure 4 | Scenarios for conserving phylogenetic diversity. Scenario 1, conservation of two distantly related species (indicated by $*$ ). Scenario 2 , conservation of two closely related species (indicated by $*$ ).

However, the situation can change if hybridization occurs within the $(\mathrm{A}, \mathrm{B})$ clade. For this case we assume that: because the species are highly specialized for their respective habitats, there is limited or no adaptive potential in each individual species, and thus without conservation of their habitat their probability for survival is zero; under conditions of environmental change hybrids will survive and reproduce, in which case there is a further probability that some hybrids will have increased adaptive potential, which will allow them to occupy altered as well as colonize new habitats.

Formally, if A (and not B) is subject to conservation efforts, let $x_{\mathrm{A}}$ be the probability that a hybrid from (A, B) will become established and survive owing to an adaptive potential greater than that of its parents. Similarly, let $x_{\mathrm{AB}}$ be the corresponding probability if both $\mathrm{A}$ and $\mathrm{B}$ receive conservation efforts (for example, by protecting the habitats they both occur in). For Scenario 1, the expected future PD is at most $\left(2 p+x_{\mathrm{A}}\right) T$, whereas for Scenario 2 it is at least $x_{\mathrm{AB}} T$ (Methods). Thus, it is now entirely possible for Scenario 2 to have higher expected future PD than Scenario 1, as this will hold whenever

$$
x_{\mathrm{AB}}>2 p+x_{\mathrm{A}}
$$

Inequality (1) will be satisfied if $p$ and $x_{\mathrm{A}}$ are sufficiently small compared with $x_{\mathrm{AB}}$.

If, in addition, hybrids can also arise within the (C, D) clade, then the corresponding condition sufficient for Scenario 2 to have higher expected PD than Scenario 1 is

$$
x_{0}+x_{\mathrm{AB}}>2 p+x_{\mathrm{A}}+x_{\mathrm{C}}
$$

where $x_{0}$ is the probability that a hybrid from $(\mathrm{C}, \mathrm{D})$ will become established if neither $\mathrm{C}$ nor $\mathrm{D}$ is conserved, and where $x_{\mathrm{C}}$ is the corresponding probability if $\mathrm{C}$ (but not $\mathrm{D}$ ) is conserved (Methods).

Although the example here is simple, in principle the calculation can be applied to any phylogenetic tree with arbitrary branch lengths, extinction probabilities, and groups of taxa capable of forming hybrids. This result complements earlier studies ${ }^{21,25}$ that have investigated how biodiversity optimization decisions that treat species independently can be modified in respect of interactions such as predator-prey relationships. Extinction risk is also unevenly distributed amongst taxa ${ }^{26,27}$. However, hybridization probabilities have the property that, in contrast to the other interaction effects previously studied, they correlate negatively with phylogenetic distance and therefore can provide contrasting PD predictions.

It is important to stress that only in instances where hybridization increases the adaptive potential does the above model apply. What is not modelled here are the conditions under which this occurs, and the contrasting situations and scenarios under which hybridization might reduce fitness ${ }^{4}$. A greater chance of adaptation is expected where a large number of hybridization events can occur between closely related species ${ }^{4}$. However, even when adaptive potential is increased, hybridization will be a double-edged sword. It has the potential to promote both long-term survival of endemic 
lineages as well as facilitate biological invasions and the replacement of endemics by exotic species ${ }^{19,28}$. As genome science delivers more detail on the nature of species, the evolutionary impact of hybridization will become clearer. A likely dilemma for future conservation efforts will be in deciding whether to work with or against hybridization and whether or not recognized species are the best units for conservation ${ }^{2,22}$.

\section{Methods}

Empirical data analyses. A map showing ice cover and potential peripheral refugia in the South Island of New Zealand during the LGM was constructed as described in Supplementary Information 1 and plotted against extant species' distributions (Fig. 1a). Accessions were genotyped using an ABI3730 sequencing protocol (Massey Genome Service) after first characterizing hot spot regions of chloroplast genome variation (Supplementary Fig. 1). These hypervariable regions were identified by Illumina sequencing and comparative analysis of chloroplast genomes for multiple accessions of $P$. fastigiatum/P. stellatum and P. enysii as also described in Supplementary Information 1. A haplotype network (Fig. 1c) was constructed for 1,463 base pairs (bp) of gene $y c f 1$ using the TCS software (see Supplementary Information 1 for details). For this analysis, indels were each recoded as single point mutations. Poly-A and poly-T sequences longer than ten bp and one site with low sequencing quality due to flanking poly-A and poly-T stretches were removed. For relaxed clock estimates of chloroplast haplotype divergence times, indels were excluded from the analyses. The alignment of concatenated sequences is given in Supplementary Information 1. A normal distribution prior of $10 \mathrm{Ma} \pm 1 \mathrm{Ma}$ was placed on the divergence time of A. thaliana from A. lyrata. An exponential distribution prior (mean $1.0 \mathrm{Ma}$, offset $=0.8 \mathrm{Ma}$ ) was used to bound the diversification age of alpine Pachycladon. Further details have been provided in Supplementary Information 1. The program DnaSPv5 (ref. 29) was used to calculate $K_{\mathrm{a}} / K_{\mathrm{s}}$ ratios in comparisons of allelic variation for glucosinolate hydrolysis genes. A $99 \mathrm{bp}$ window and step size of $9 \mathrm{bp}$ was used. Gene-wide $K_{\mathrm{s}}$ values were used to avoid the problem of $K_{\mathrm{s}}=0$ within individual windows. For statistical tests of hybridization we used: JML (ref. 11); the species tree from Joly et al. (manuscript in preparation); and concatenated sequences of chloroplast genes or glucosinolate hydrolysis genes. Details of computational analyses have also been included in Supplementary Information 1.

Phylogenetic diversity. For our model of phylogenetic diversity loss accompanying possible hybridization within the $\mathrm{AB}$ clade, the expression $\left(2 p+x_{\mathrm{A}}\right) T$ in Scenario 1 arises from writing the random variable $P D$ as $P D=X_{1} T+X_{2} T$, where $X_{1}$ and $X_{2}$ are 0,1 random variables with $X_{1}=1$ if either A survives or an (A, B) hybrid becomes established and survives, and $X_{2}=1$ if $C$ survives. Then the expected PD is $\operatorname{Pr}\left(X_{1}=1\right) T+\operatorname{Pr}\left(X_{2}=1\right) T$ and because $\operatorname{Pr}\left(X_{1}=1\right) \leq p+x_{\mathrm{A}}$ by Boole's inequality, and $\operatorname{Pr}\left(X_{2}=1\right)=p$, the expected PD is no more than $\left(2 p+x_{\mathrm{A}}\right) T$. For Scenario 2 we have $P D=X T$, where $X$ is the 0,1 random variable that equals 1 precisely when at least one of the following three events occur: A survives, B survives, or the event $(\mathrm{H})$ that a hybrid of $\mathrm{A}$ and $\mathrm{B}$ becomes established and survives. Thus, the expected PD in this case is $\operatorname{Pr}\left(X_{1}=1\right) T \geq \operatorname{Pr}(H) T=x_{\mathrm{AB}} T$. Thus, if Inequality (1) holds, Scenario 2 will have higher expected PD than Scenario 1.

This analysis extends to the setting where hybrids can also arise within the $(\mathrm{C}, \mathrm{D})$ clade by noting that the expected $\mathrm{PD}$ in Scenario 2 is at least $x_{0} T+x_{\mathrm{AB}} T$ whereas for Scenario 1 it is at most $\left(p+x_{\mathrm{A}}\right) T+\left(p+x_{\mathrm{B}}\right) T$ so when Inequality (2) holds, Scenario 2 will again have a higher expected PD than Scenario 1.

Received 3 May 2013; accepted 16 September 2013; published online 3 November 2013

\section{References}

1. Hoffmann, A. A. \& Sgrò, C. M. Climate change and evolutionary adaptation. Nature 470, 479-485 (2011).

2. Bálint, M. et al. Cryptic biodiversity loss linked to global climate change. Nature Clim. Change 1, 313-318 (2011).

3. Anderson, J. T., Panetta, A. M. \& Mitchell-Olds, T. Evolutionary and ecological responses to anthropogenic climate change: Update on anthropogenic climate change. Plant Physiol. 160, 1728-1740 (2012).

4. Abbott, R. et al. Hybridization and speciation. J. Evol. Biol. 160, 229-246 (2013).

5. Muhlfeld, C. C. et al. Hybridization rapidly reduces fitness of a native trout in the wild. Biol. Lett. 5, 328-331 (2009).

6. Rieseberg, L. H. Evolution: Replacing genes and traits through hybridization. Curr. Biol. 19, R119-R122 (2009).

7. Arnold, M. L. Transfer and origin of adaptations through natural hybridization: Were Anderson and Stebbins right? Plant Cell 16, 562-570 (2004).

8. The Heliconius Consortium, Butterfly genome reveals promiscuous exchange of mimicry adaptations among species. Nature 487, 94-98 (2012).
9. Heenan, P. B. \& Mitchell, A. D. Phylogeny, biogeography and adaptive radiation of Pachycladon (Brassicaceae) in the mountains of South Island, New Zealand. J. Biogeograph. 30, 1737-1749 (2003).

10. Joly, S., Heenan, P. B. \& Lockhart, P. J. A Pleistocene inter-tribal allopolyploidization event precedes the species radiation of Pachycladon (Brassicaceae) in New Zealand. Mol. Phylogenet. Evolut. 51, 365-372 (2009).

11. Joly, S. JML: Testing hybridization from species trees. Mol. Ecol. Res. 12, 179-184 (2012).

12. Zhang, Z., Ober, J. A. \& Kliebenstein, D. J. The gene controlling the quantitative trait locus EPITHIOSPECIFIER MODIFIER1 alters glucosinolate hydrolysis and insect resistance in Arabidopsis. Plant Cell 18, 1524-1536 (2006).

13. Lambrix, V., Reichelt, M., Mitchell-Olds, T., Kliebenstein, D. J. \& Gershenzon, J. The Arabidopsis epithiospecifier protein promotes the hydrolysis of glucosinolates to nitriles and influences Trichoplusia $n i$ herbivory. Plant Cell 13, 2793-2807 (2001).

14. Agee, A. E. et al. Modified vacuole phenotype1 is an Arabidopsis myrosinase-associated protein involved in endomembrane protein trafficking. Plant Physiol. 152, 120-132 (2010).

15. Voelckel, C. et al. Transcriptional and biochemical signatures of divergence in natural populations of two species of New Zealand alpine Pachycladon. Mol. Ecol. 17, 4740-4753 (2008).

16. Wittstock, U. \& Burow, M. Glucosinolate breakdown in Arabidopsis: Mechanism, regulation and biological significance. Arabidopsis Book 8, e0134 (2010).

17. Voelckel, C., Gruenheit, N., Biggs, P., Deusch, O. \& Lockhart, P. J. Chips and tags suggest plant-environment interactions differ for two alpine Pachycladon species. BMC Genom. 13, e322 (2012).

18. Nadachowska-Brzyska, K., Zieliński, P., Radwan, J. \& Babik, W. Interspecific hybridization increases MHC class II diversity in two sister species of newts. Mol. Ecol. 21, 887-906 (2012).

19. Abi-Rached, L. et al. The shaping of modern human immune systems by multiregional admixture with archaic humans. Science 334, 89-94 (2011).

20. Benton, M. J. The red queen and the court jester: Species diversity and the role of biotic and abiotic factors through time. Science 323, 728-732 (2009).

21. Moulton, V., Semple, C. \& Steel, M. Optimizing phylogenetic diversity under constraints. J. Theoret. Biol. 246, 186-194 (2007).

22. O’Brien, S. J. \& Mayr, E. Bureaucratic mischief: Recognizing endangered species and subspecies. Science 251, 1187-1188 (1991).

23. Allendorf, F. W., Leary, R. F., Spruell, P. \& Wenburg, J. K. The problems with hybrids: Setting conservation guidelines. Trends Ecol. Evol. 16, 613-622 (2001).

24. Weitzman, M. L. The Noah's Ark problem. Econometrica 66, 1279-1298 (1998).

25. Witting, L., Tomiuk, J. \& Loeschecke, V. Modelling the optimal conservation of interacting species. Ecol. Model. 125, 123-144 (2000).

26. Purvis, A., Agapow, P. M., Gittleman, J. L. \& Mace, G. M. Nonrandom extinction and the loss of evolutionary history. Science 288, 328-330 (2000).

27. Thuiller, W. et al. Consequences of climate change on the tree of life in Europe. Nature 470, 531-534 (2011).

28. Ryan, M. E., Johnson, J. R. \& Fitzpatrick, B. M. Invasive hybrid tiger salamander genotypes impact native amphibians. Proc. Natl Acad. Sci. USA 106, 11166-71 (2009).

29. Librado, P. \& Rozas, J. DnaSP v5: A software for comprehensive analysis of DNA polymorphism data. Bioinformatics 25, 1451-1452 (2009).

\section{Acknowledgements}

N.G. and O.D. were supported by Postdoctoral Fellowships from the German Academic Exchange Service (DAAD). C.V. was a recipient of an Alexander von Humboldt Feodor Lynen Research Fellowship. This work was initiated with financial support from the New Zealand Marsden Fund and received additional project funding from Massey University. P.J.L. and M.S. contributed to this work while New Zealand Royal Society James Cook Fellows. We thank B. Martin, S. Joly and K. Sluis (Illumina) for their support and encouragement, and V. Symonds for constructive comments.

\section{Author contributions}

M.B., N.G., O.D., C.V., P.B.H. and P.J.L. designed the experiments and conducted most analyses. P.A.M. and O.K. provided technical support. The authorship order reflects relative contributions. J.W.L. designed and conducted the recombination breakpoint test. P.J.L. developed the conjecture, and M.S. the mathematical model described in the manuscript.

\section{Additional information}

Supplementary information is available in the online version of the paper. Reprints and permissions information is available online at www.nature.com/reprints. Correspondence and requests for materials should be addressed to M.B.

\section{Competing financial interests}

The authors declare no competing financial interests. 